mann. Beide müssen aber eben die GUSbox nutzen - also über dieselbe Kommunikationstechnik verfügen. Auch wer den Weg über KV-Connect wählt, sollte daher sicherstellen, dass der Empfänger ebenfalls an KV-Connect angebunden ist. Werden Mail-Lösungen wie die GUSbox EMail genutzt, benötigt der Empfänger hingegen nur eine E-Mail-Adresse.

Der Versand an sich lässt sich unkompliziert aus der Praxissoftware heraus initiieren, wie das Beispiel Duria zeigt. Dort stehen Ärzten als Übermittlungsweg das D2D-Verfahren, KV-Connect und KOM-LE der gematik zur Verfügung. Alle Verfahren sind in den normalen Praxis-EDV-Workflow integriert, werden also direkt innerhalb der Arzt-software bereitgestellt. Die E-Arztbriefe lassen sich dabei mit dem E-Heilberufsausweis signieren. Die Übertragung der Daten läuft über einen verschlüsselten Kanal es wird ein sogenanntes Virtual Private Network (VPN) genutzt. Zusätzlich ist laut Duria das auszutauschende Doku- ment von Anfang bis Ende der Kommunikation verschlüsselt.

\section{Alle Daten werden verschlüsselt}

Die sichere Übertragung der Daten ist dabei Standard bei allen Softwareanbietern: Bei den Softwarelösungen der medatixx werden für den möglichen Versand via D2D oder den Versand über den medatixx-Standard comdoxx Verschlüsselungsverfahren eingesetzt, die durch datenschutzrechtliche Gutachten bestätigt wurden. Der Arztbriefversand via comdoxx steht dabei allen Ärzten, die medatixx-Systeme nutzen, zur Verfügung - ist laut dem Softwarehaus aber zusätzlich in fünf Fremdsystemen integriert, so etwa in Duria. Alle Ärzte könnten jedoch in den medatixx-Systemen ebenso Arztbriefe per E-Mail versenden. Der Vorteil der etablierten Systeme wie comdoxx oder D2D sei jedoch, dass zum Teil sogar eine automatische Datenübertragung an ausgewählte Praxen stattfinde, heißt es aus dem Haus medatixx. Voraussetzung sei ledig- lich eine einmalige Bestätigung der teilnehmenden Praxis, dass sie in der Lage ist, derartige Nachrichten zu empfangen.

Bei den Systemhäusern der CompuGroup Medical (CGM) läuft der sichere, verschlüsselte Versand entweder über KVConnect oder über ein eigens entwickeltes System, das CGM Connect. Bei der Frey ADV GmbH haben die Praxen - wie skizziert - die Wahl zwischen GUSbox, KVConnect und KV-Safemail oder einem via sicheren Passwort verschlüsselten E-MailVersand. Während Duria aber etwa auf die E-Arztbrief-Technologie der KVen setzt, kommt bei den Häusern der CompuGroup (Albis, M1 Pro, Medistar, TurboMed) der VHitG-Arztbrief - und damit der Standard aus dem Bundesverband Gesundheits-IT - zum Einsatz. Interessant ist, dass auch Kliniken, bei denen CGM Jesajanet mit E-Arztbrief-Modul verwendet wird, eine direkte Austauschmöglichkeit mit den Praxen haben. Laut CGM funktioniert der Versand über Systemgrenzen hinweg.

RebekkaHöhl

\title{
E-Health-Gesetz: Halbherzige Vorgaben für einen professionellen Datenaustausch
}

\author{
Interoperabilität? An der wird es auch mit dem E-Health-Gesetz \\ noch fehlen, kritisiert der Bundesverband Gesundheits-IT. Denn \\ der Gesetzgeber geht das Problem falsch an.
}

Mit dem Entwurf zum E-Health-Gesetz ist genau das eingetreten, was man aus Industriesicht - aber eigentlich auch aus Anwender- und Arztsicht - hatte verhindern wollen: Die in dem Entwurf so hoch gehaltene Interoperabilität zwischen den Praxis- und Kliniksystemen wird gerade nicht gefördert. „Im E-Health-Gesetz werden zwei Themen vermischt: Die tatsächliche Interoperabilität - also der fallund behandlungsbezogene Datenaustausch - und die Datenportabilität beim Systemwechsel“, sagt Jens Naumann, Vorstandsmitglied beim Bundesverband Gesundheits-IT (bvitg). Zusätzlich hat es der Gesetzgeber versäumt, in seinem Entwurf für alle an der Gesundheits-IT Beteiligten klare Vorgaben festzuschreiben. Naumann: „Der Gesetzgeber sagt lediglich, dass es ein Interoperabilitätsverzeichnis geben soll." Er definiere aber nicht, wie genau ein elektronischer Arztbrief (E-Arztbrief) oder andere Dokumente auszuse- hen haben. Ein strukturiertes Dokument zu erstellen und in ein anderes System zu übertragen ist schon seit langem möglich. „Es gibt aber 50 verschiedene Wege dies zu tun“, schildert Naumann das eigentliche Problem. Und genau dies geschieht auch im Praxis- und Klinikalltag wie alleine das Beispiel des E-Arztbriefes zeigt: Es gibt das D2D-Verfahren, das künftig vom KV-Connect-Brief abgelöst wird, KOM-LE der gematik, den VHitG-Arztbrief, E-Arztbrief-Standards einzelner Berufsverbände und dann auch noch regional selbst gestrickte Standards innerhalb von Praxisverbünden. Naumann: „Jeder greift in die große Kiste der IT-Standards und sagt, so will ich es haben." Laut Naumann wäre es daher längst an der Zeit, dass man sich auf eine einheitliche Sprache (Syntax) und eine einheitliche Semantik - also was etwa der E-Arztbrief enthalten soll - einige. Ebenfalls wichtig sei eine Regelung, wie signiert und verschlüsselt und auf welchem Weg die Daten übertragen werden. Darin liegt auch ein Hauptkritikpunkt des bvitg am Entwurf zum EHealth-Gesetz. Der Verband wünsche sich, dass der Gesetzgeber festschreibt, dass es ein Interoperabilitätsverzeichnis gibt und dass er definiert, dass beispielsweise ein E-Arztbrief immer im Schema HL7 geschrieben wird, mit dem E-Heilberufsausweis zu zertifizieren ist etc. - zumindest wenn Briefe innerhalb des GKVSystems elektronisch übertragen und gefördert werden.

Naumann sieht aber noch ein weiteres Problem, das es zu lösen gilt: die Nutzenargumentation gegenüber den Ärzten. In den Praxen würden die Prozesse an sich laufen. Ärzte nutzen einfach Word-Vorlagen für ihre Briefe und senden diese wie gehabt per Fax raus. Der tatsächliche Vorteil des E-Arztbriefes liege in der intelligenten Verarbeitung der Daten im System, erläutert Naumann. „Ein strukturierter Brief sendet mir die Diagnose so, dass ich die Daten direkt in meiner Software verarbeiten kann." Und genau deshalb sind wieder einheitliche Standards wichtig, damit jeder Brief in jeder beliebigen Praxissoftware gleich einlaufen kann. Naumann: „Es müssen aber auch pragmatische Lösungen für die Signatur geschaffen werden." Rebekka Höhl 